\title{
ThermoLabAnimal - A high-throughput analysis software for non-invasive thermal assessment of laboratory mice
}

\author{
Nuno Henrique Franco ${ }^{a, b, 1}$, Ana Gerós ${ }^{b, c, d, 1}$, Liliana \\ Oliveira $^{a, b}$, I. Anna S. Olsson ${ }^{a, b}$, Paulo Aguiar ${ }^{b, c,}$
}

\section{These authors contributed equally to this paper.}

a. IBMC - Instituto de Biologia Molecular e Celular, Universidade do Porto,

b. i3S - Instituto de Investigação e Inovação em Saúde, Universidade do

c. INEB - Instituto Nacional de Engenharia Biomédica, Universidade do

d. FEUP - Faculdade de Engenharia, Universidade do Porto,

*Originally published in Physiology \& Behavior 207 (2019) 113-121

\section{ABSTRACT}

Body temperature changes in laboratory mice are often assessed by invasive and stressful methods, which may confound the measurement. Infrared thermography is a possible non-invasive alternative, but the cost of standard thermal cameras, lack of dedicated software for biomedical purposes, and labour-intensiveness of thermal image analysis have limited their use. An additional limitation lies on the scarcity of research on the causing factors of differences between body surface and core body temperature. We propose a method for automatic assessment of mean body surface temperature in freely-moving mice, using dedicated software for thermal image analysis. While skin surface temperature may not necessarily be linearly correlated with core body temperature (in itself an imprecise concept), under standardized environmental conditions, such as those in which laboratory animals are kept, mean body surface temperature can provide useful information on their thermal status (i.e. deviations from normothermia, namely hypo- and hyperthermia). We developed a publicly available software that includes an imaging analysis workflow/algorithm for automatic segmentation of the pixels associated with the animal from the pixels associated with the background, removing the need for manually defining the area of analysis. A batch analysis mode is also available, for automatic and high-throughput analysis of all image files located in a folder. The software is compatible with the most widespread thermal camera manufacturer, 'FLIR Systems', as well as with the low-cost 'Thermal Expert TE- $\mathrm{Q}_{1}$ ' miniaturized high-resolution thermal camera used for this study. Furthermore, the software has been validated in a mouse model expressing nontransient hypothermia, where the thermal analysis results were compared with readings from implanted thermo-sensitive passive integrated transponders tags. Thermography allows for thermal assessment of laboratory animals without the effect of handling stress on their physiology or behaviour. Our automatic image analysis software also removes observer errors and bias, while speeding up the data processing. 


\section{Introduction}

Body temperature variation can provide valuable information in animal-based biomedical research. An increasingly used method for monitoring thermal changes in laboratory animals is infrared thermography (IRT), which allows contactless estimation of body surface temperature variations, with several applications in research [1-7]. This approach prevents the scientific and welfare impact of more invasive methods for thermal assessment. The use of rectal or infrared thermometers directly affects the temperature readout, due to a core temperature rise resulting from a hyperthermic stress response mediated by the sympathetic-adrenal and the hypothalamicpituitary-adrenal (HPA) axes [8,9]. This is accompanied by a sympathetically-mediated vasoconstriction, resulting in a transient drop in the extremities (particularly in the tail), which rebounds by the warming from vasodilation for core heat dissipation $[10,11]$. This response is moreover heightened by repeated handling $[12,13]$ and can even be elicited prior to handling, in response to alarm calls from handled animals [12-14]. Surgically implanted sensors can be read from a distance [15]; however, these methods are not non-invasive in themselves. Sensor implantation requires surgery under general anaesthesia, and adds considerably to workload, having also an impact on animal welfare [16-18].

The use of IRT is not new in veterinary research and practice [19-21] but its use with laboratory animals has been limited, probably as a result of the cost (in the thousands of Euros range) and bulkiness of most IRT cameras, which are incompatible with measurements in small rodent cages, in combination with the lack of dedicated analysis software.

To the best of our knowledge, there are no infrared thermography imaging software dedicated/tailored for laboratory mice, and researchers often rely on general purpose and proprietary software for direct pixel (temperature) readouts (e.g. Fluke SmartView, FLIR Tools, or Testo IrSoft). Despite these difficulties, IRT technology has been proven useful in laboratory animal science in identifying housing problems [7], following neonatal development [22], identifying stress [23] and monitoring infections $[24,25]$, among other applications. In a previous study, we found IRT readout of mean body surface temperature (MBST) to be a more reliable parameter for non-invasive assessment of surface temperature variation in freely-moving mice than either maximum eye or tail temperature [1]. This parameter has also been successfully used in previous studies in mice $[2,6,26,27]$, as well as in other species, including rabbit pups [4,28], chickens [29] and animals in the wild $[30,31]$. The inherently limited accuracy and noise of thermal cameras, the inhomogeneity in the animals' surface emissivity, along with the presence of other interfering heat sources, render temperature measurements based on a single spot error-prone. A better approach may be to average larger body surface areas, which are relatively isothermal if the ambient temperature remains unchanged, as is the case in environmentally controlled laboratory animal facilities. In a previous study [1], we assessed MBST by manually defining regions of interest (ROIs) corresponding to the contour of each animal, in each image. Image analysis was then performed on the ROls to extract, for example, mean thermal values. This process was found to be laborious, time-consuming, userdependent, error-prone and difficult to perform consistently.

In response to these challenges, we hereby present a dedicated computational tool developed for automatic assessment of MBST from a virtually unlimited number of thermal images. This new software greatly facilitates surface temperature quantification of freely-moving mice. The software uses image segmentation algorithms that remove background heat and focus the analysis in specific ROls. Further, it allows both individual thermal image screening and batch analysis of thermal images. The type of image analysis to perform, single or batch, as well as other options and features, are chosen using a simple and intuitive graphical user interface (GUI). The software reads thermal 
images saved as .CSV ("comma separated values") files, a format to which most thermal cameras can save images directly, or convert to, using the camera's software.

We validated our computational tool and analysis protocol in a mouse model of acute septic shock by intraperitoneal injection of a high dose of lipopolysaccharide (LPS), of which a pathophysiological hallmark is quick-onset, pronounced hypothermia. The aim was to assess whether MBST could reliably inform on body temperature changes in this model, as compared with data from the well-established method of subcutaneously-implanted thermo-sensitive PIT-tags. Subcutaneous PIT tags are a widely used method for non-invasive measurements but have many limitations, as presented before. The objective is not to compare the IRT methodology itself with PIT tag measurements, but instead to emphasise the advantages that ThermoLabAnimal brings when using IRT. In compliance with the 3 Rs principle of Reduction, measurements were obtained as additional data from an already scheduled experiment, in an ongoing project in our institution. The software can be downloaded from https://github.com/ThermoLabAnimal

\section{Materials and methods}

\subsection{Thermal cameras}

A miniaturized $(47 \mathrm{~mm} \times 25 \mathrm{~mm} \times 25 \mathrm{~mm})$ 'Thermal Expert TE- $\mathrm{Q}_{1}$ ' (i3 Systems, Korea) thermal camera was used in all animal thermal imaging experiments. According to the manufacturer, it has a $384 \times 288$ pixels resolution, thermal sensitivity below $50 \mathrm{mK}$ and $\pm 3^{\circ} \mathrm{C}$ or $\pm 3 \%$ accuracy (depending on environmental conditions). To assess the camera's accuracy, we tested the accuracy of the low-cost using a black body radiation source (see Supplementary material).

The camera was placed $30 \mathrm{~cm}$ above the cage, alongside an RGB Microsoft LifeCam HD-3000 camera (Redmond, Washington, USA) to allow visual identification of the animals, with the camera's optical axis perpendicular to the cage floor. Both cameras were connected via OTG USB to an ASUS T101Ha computer (running OS Windows 10) and operated via the Thermal Expert proprietary software (version 1.7.0). Emissivity was set to 0.95 .

All animal thermal measurements were carried out after an equipment warm-up period of 30 minutes (i.e. the camera was turned on 30 minutes before the first animal measurement, and continued to operate continuously until completing the last measurement).

With the purpose of widening and validating the compatibility of this computational tool, we made it capable of reading and analysing files generated by FLIR cameras (the most broadly used thermal camera brand). Compatibility was assessed using images of freely-moving mice taken by a 'FLIR E6o' (320×240 pixels resolution, <50mK thermal sensitivity and $\pm 2^{\circ} \mathrm{C}$ or $\pm 2 \%$ accuracy; FLIR Systems, USA), after being converted to .CSV using the FLIR proprietary software (FLIR Tools)

\subsection{Software development}

A dedicated thermal imaging analysis software, named ThermoLabAnimal, was developed in MATLAB R2018a (The MathWorks Inc., USA) and took advantage of the advanced image analysis algorithms available in MATLAB's Image Processing Toolbox. In order to automatically segment the animals' bodies, and separate the background from foreground pixels, a global thresholding method

(1) 
was used, where the threshold value was calculated in order to minimize the intra-class variance (Otsu's method). The segmentation mask was corrected with the removal of small patches (associated in general with urine spots), by eliminating all the components (using an eight neighbours connectivity) with an area smaller than a defined (but user modifiable) value. This corrected mask was then used to perform the thermal analysis statistics exclusively in pixels associated with the animal(s). The analysis workflow also included an optional step for the identification of multiple animals in the mask. In situations where animals are not juxtaposed, a connected-components analysis can be performed and the mask is segregated into individual components (associated with the individual animals).

The GUI for the software was developed using MATLAB's graphical interfaces development environment (GUIDE). Import capabilities were included providing the ability to load files with thermal data from both 'Thermal Expert' and FLIR Systems' cameras. ThermoLabAnimal was developed for compatibility with both Microsoft Windows and Apple operating systems.

\subsection{Animals}

To validate the ThermoLabAnimal software, we obtained thermal images from $C_{57} \mathrm{BL} / 6$ mice undergoing procedures in a different project (DGAV license o09951), which involved large-dose LPS injection via the intraperitoneal route. No additional intervention, other than the thermal imaging, was imposed on the animals for the purpose of the present study. We present pooled data from nine mice from three of the experimental groups, the control group and two of the experimentally treated groups for which surface temperature variation did not differ significantly from that of control mice (one-way ANOVA, $d f=2, s s q=2.120, F=1.426, p=0.244$ ). To preserve confidentiality of yet unpublished results, treatments are not disclosed. Four 'control' mice were housed with one mouse from an 'experimental' group in a 'type II' (dimensions: $268 \times 215 \times 141 \mathrm{~mm}$, floor area: $370 \mathrm{~cm} 2$ ) cage, whereas the other seven mice were housed together in a 'type III' (dimensions: $425 \times 276 \times 153 \mathrm{~mm}$, floor area: $820 \mathrm{~cm} 2$ ) cage (Tecniplast, Italy). All cages contained corncob bedding (LBS serving Biotechnology, United Kingdom), absorbent paper (Renova, Portugal) for nesting material, and a cardboard tube (LBS serving Biotechnology, United Kingdom). Mice had access to Teklad Harlan $2014 \mathrm{~S}$ (Envigo, United Kingdom) chow and tap water ad libitum. Room temperature was maintained at $20-24^{\circ} \mathrm{C}$ with a relative humidity of $45-65 \%$. Mice were housed under a $12: 12$ hour dark/light cycle with lights on between $08: 00 \mathrm{~h}$ and $20: 00 \mathrm{~h}$.

Fig. 1. Graphical user interface (GUI) of the ThermoLabAnimal software. a) The main GUI provides simple access to the software tools/features, namely the option between single image analysis and batch analysis. b) After loading a thermal image in single image analysis, the user can inspect the image using standard visualization tools (zoom, read specific pixel values, etc.). c) The automatic segmentation and thermal background elimination algorithms allow isolation of the pixels associated with the mice. A histogram for surface temperature distribution of all mice in the frame, and a three-dimensional representation of the temperature map, integrate the analysis outputs 
a)

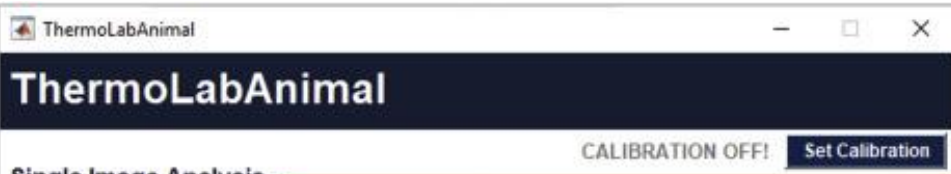

Single Image Analysis

CALIBRATION OFF I

Select file: C.DataiThermolmagesi $\ldots$

1) ALL ANIMALS POOLED

Start analysis: START

2) INDIVIDUAL ANIMALS

Start analysis: START

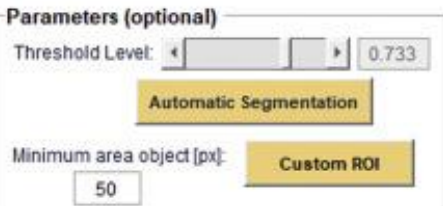

Batch Analysis

Select folder:

Start analysis and save:

$\square$ Save also background info

Ana Gerós \& Paulo Aguiar [paubaguiareineb.up.pl] 2018

b) If Figue: Singiel image Anatygis - coliormap

file fad Vien Imet Toch Derbitep windaw Help

O日日)

Colormap - colorbar with temperature in ${ }^{\circ} \mathrm{C}$

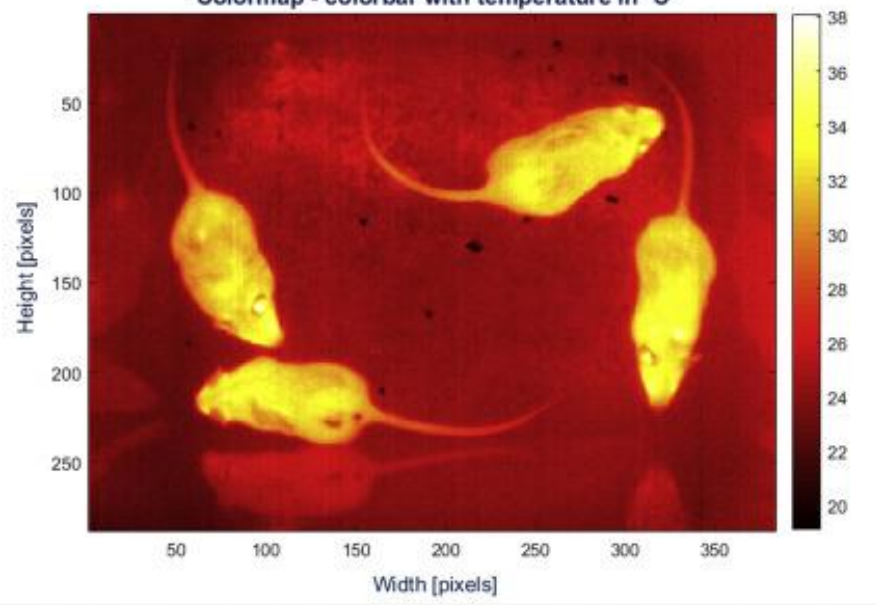

c) Th figure: Single image Anatypis - colormap

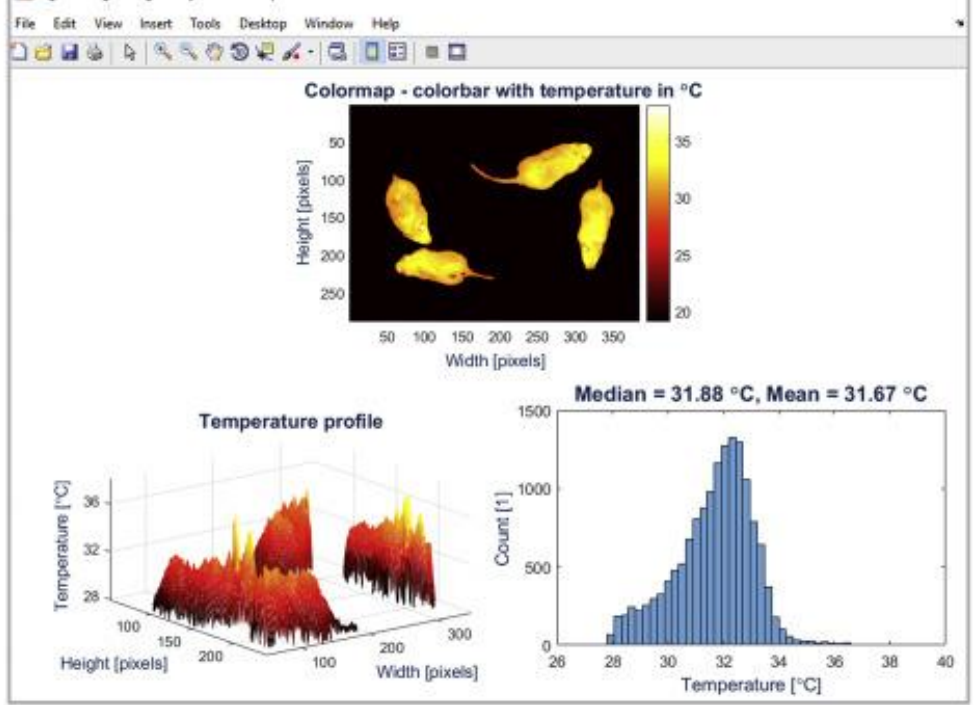




\subsection{Experimental protocol}

The mice were subcutaneously implanted with a Biotherm thermosensitive passive integrated transponder (PIT) tag, using a 12-gauge needle, under short-term $(<5$ minutes) isoflurane anaesthesia (administered with 1 bar of oxygen at $5 \%$ concentration for induction, and with 0.2 bar of oxygen at $2 \%$ concentration for maintenance). PIT tags were read by a Destron Fearing GPR+ handheld reader (with a reading range between $33-43^{\circ} \mathrm{C}$ ). The puncture site was sealed with surgical glue, but in two of the animals the tags were nonetheless exteriorized and found the following day on the cage bedding. These animals, as well as a third which maintained normothermia throughout the experiment (possibly due to error in administration of LPS), were removed from the study. Hence, subcutaneous and mean body surface temperature of a total of $\mathrm{N}=9$ animals (three per each of the treatment groups) was monitored. One week after PIT tag implantation, animals were marked in the tail (by coloured marker) for fast visual identification, and injected intraperitoneally with $12.5 \mathrm{mg} / \mathrm{kg}$ of LPS from Escherichia coli O111:B4 (Sigma Aldrich). Thermal images were collected during a period of 6 hours: every 10-20 minutes post-injection during the first hour and a half (namely at (at 0:20, $0: 32,0: 45,0: 58,1: 12,1: 22,1: 36,1: 48$ hours post-injection), and then at more spaced intervals (namely at $2: 28,3: 00,4: 42,5: 15,5: 44,6: 13$ hours post injection). At each time-point, the cage lid was open, the nesting material and cardboard tube were gently removed and three images were taken of the group, when all animals had all four paws on the cage floor. At each time-point, image collection of the group of animals took about 1 min to perform. At $t=4.67 \mathrm{~h}$, mean subcutaneous temperature reached $33.3^{\circ} \mathrm{C}$, and cages were placed over a warm heating pad.

\subsection{Statistics}

Significant changes between time-points were assessed by paired-samples t-test., with a HolmBonferroni correction for multiple comparisons. The threshold for significance was set at $\mathrm{p}<0.01$.

Temperature decrease was expected to be very pronounced, following LPS challenge. Through a power calculation (using G-Power software) for a one-sided matched-pairs test with $\alpha=0.01$, a power of $80 \%$, and a standardized effect size Cohen's $\mathrm{d}=2$ (mean difference at least as large as two standard deviations) a total sample size of six mice was deemed sufficient. Our sample size of $\mathrm{N}=9$ would allow identifying smaller differences, with the same statistical power, for the same significance level. The IBM SPSS Statistical package (version 25) was used.

\section{Results}

\subsection{ThermoLabAnimal graphical user interface}

The computational tool ThermoLabAnimal runs on both Microsoft Windows and Apple's operating systems, and has a user-friendly graphical user interface (GUI) allowing an easy workflow of importing the thermal images, analysing the data and saving the results (Fig. 1). Two modes of analysis are available in the main GUI (Fig. 1a): single image and batch analysis (performed on all files in a specified folder). In the single image analysis mode, the user can select a thermal image in .CSV format. The segmentation algorithm automatically creates a mask for the animals, separating foreground pixels (animal) from background pixels. From the single image analysis, two figures are generated. The first figure shows an unaltered false-colour thermal image reconstructed from the .CSV file, along with the thermal colour legend (Fig. 1b). The second figure is composed of three 
elements (Fig. 1C): i) a reconstructed image with the automatic mask applied to eliminate the background; ii) a 3 -dimensional temperature map ( $\left.x, y,{ }^{\circ} T\right)$, which can be freely rotated; and iii) a histogram of temperatures in the mask (animal surface). All animals in a cage can be considered an experimental unit or, alternatively, each can be analysed individually (Fig. 2). If in the thermal image the animals are not overlapping, ThermoLabAnimal can automatically segment each one individually (Fig. 2a) and present separate thermal analysis for each animal (Fig. 2b). All output figures are interactive and can be exported.

While the automatic segmentation algorithm works in most cases, mask fine-tuning may be necessary in some conditions (Fig. 3). For this purpose, ThermoLabAnimal provides manual adjustment of the threshold with immediate visualization feedback. Furthermore, in order to eliminate odd detections (e.g. urine spots), the software allows automatic removal of all blobs smaller than a user-defined number of pixels, for both single and batch image analyses (Fig. 3a). To keep ThermoLabAnimal as versatile as possible, users can also perform the thermal analysis of a userdefined ROI (Fig. 3b). This feature can be used to analyse local temperatures at specific anatomical regions (e.g. eye, tail, wounded or inflamed region, etc.).

The batch analysis mode allows automatic and high-throughput thermal data analysis from all the image files located in a specified folder. The batch analysis produces two outputs. The first is a single spreadsheet document, listing the mean and median MBST of all animals identified in each thermal picture. The second is, for each .CSV file, an automatically-generated composite image similar to the output of single image analysis with a false-colour thermal image, a thermal histogram, and a 3 -dimensional temperature map $\left(x, y,{ }^{\circ} \mathrm{T}\right)$. In the batch analysis mode, the user can choose to also export information about the background of each image in the folder. In parallel, a similar composite image is generated, containing information about the background, obtained using the counter-mask that separates the foreground and background pixels.

\subsection{Software output}

The software successfully recognized each individual animal from their thermal background (Fig. 1), in every image, providing a histogram for the distribution of group mean and median body surface temperature, along with a fully-adjustable 3D map (pixel coordinates vs temperature). The thermal profile of specific areas could be obtained by defining a contour/ROI (Fig. 3). Using the batch analysis function, a dataset of 1384 . CSV files were automatically analysed, obtaining MBST in a standardized way, thus eliminating the possibility of unwanted variation and operator bias. The output was a single spreadsheet file with values for group mean and median temperature for every .CSV image, as well as an automatically generated composite image for each file analysed. Even when surface temperatures differed considerably between animals in the same frame, the software was successful in highlighting each animal in the frame. However, in the extreme cases when the MBST of some animals decreased to the point where their thermogram blended with the background, the detection threshold had to be manually adjusted for those images.

\subsection{High-dose LPS challenge}

Immediately following a high-dose LPS challenge, there was a small subcutaneous temperature rise in both the PIT tag readout and MBST, yet not found to be significant (Fig. 4). This was followed 
by a steady decrease in subcutaneous temperature, along with a faster and more pronounced decrease in MBST, which remained low until decreasing further at $t=5.25 \mathrm{~h}$. At $t=4.67 \mathrm{~h}$ mean subcutaneous temperature was estimated as $33.3^{\circ} \mathrm{C}$, with most animals (six out of nine) falling below the reading range of the PIT tag reader of $33.0^{\circ} \mathrm{C}$ (and registered as $32.9^{\circ} \mathrm{C}$ ). At $\mathrm{t}=4.67 \mathrm{~h}$ cages were placed over a warm heating pad, in compliance with institutional animal welfare guidelines. This pattern was consistent for all three groups (Fig. 4a). The aggregated information from all groups is presented in Fig. $4 \mathrm{~b}$.

Fig. 2. The software is also capable of individualized thermal analysis, as long as the animals are not overlapping. a) Using the "Individual Animals" analysis option, the software detects and labels each animal. However, animal identity is not maintained between images. b) For each separated animal in the image, the software outputs a dedicated thermal analysis window.

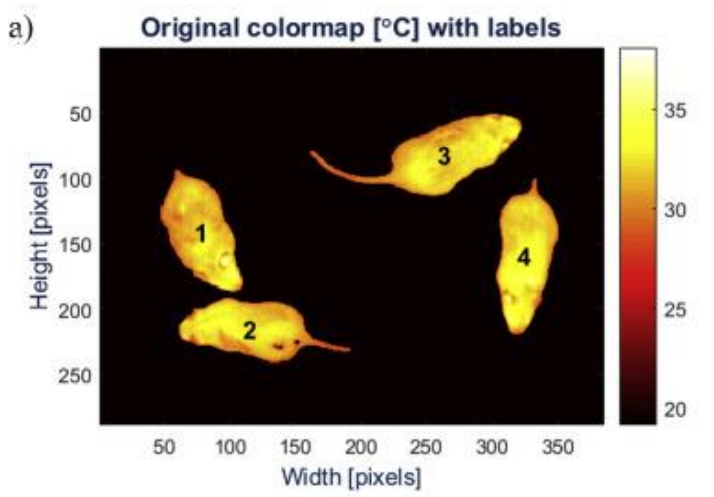

Original image with colored objects and labels

b)

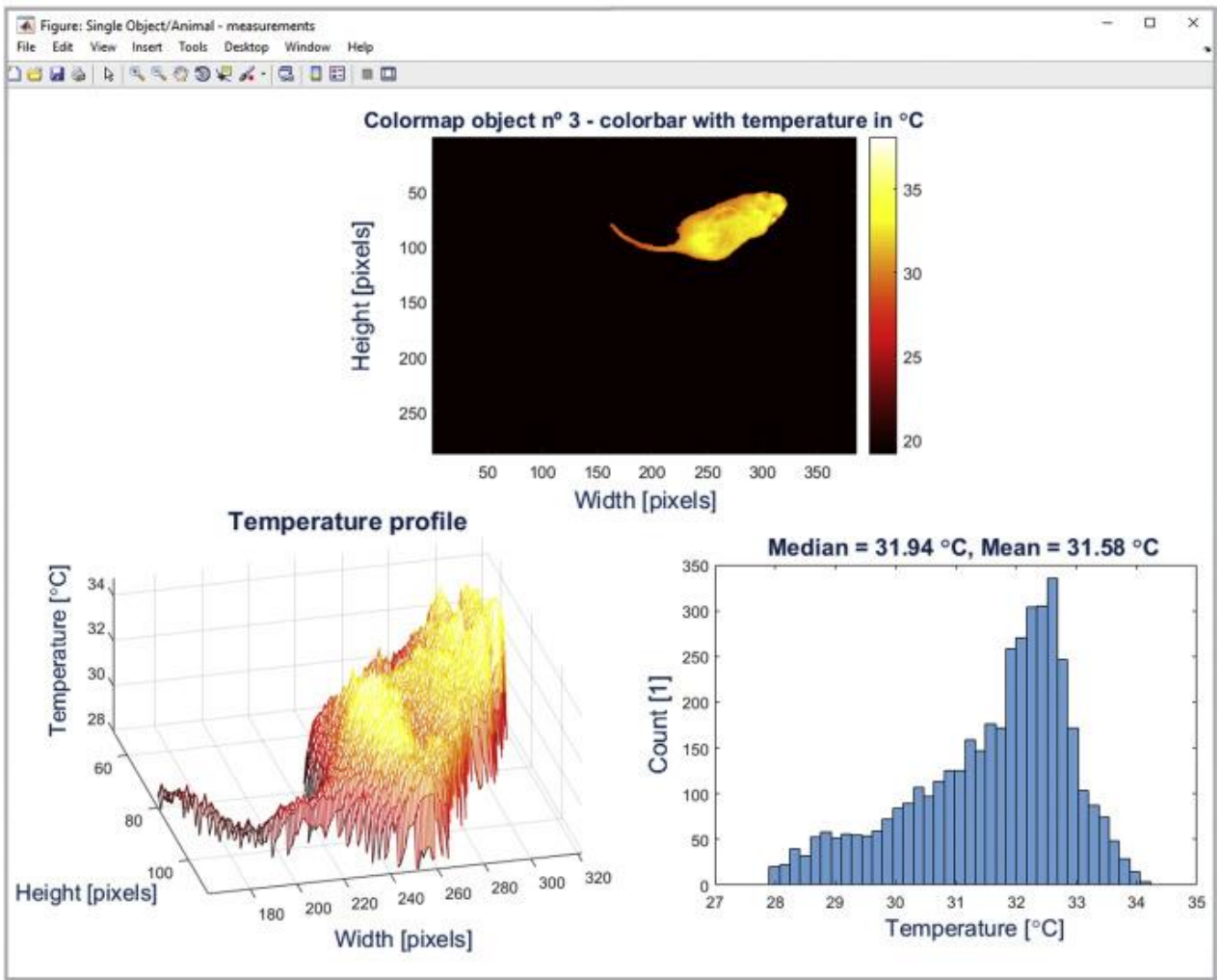


Fig. 3. Additional tools in ThermoLabAnimal. a) To eliminate odd occurrences, such as urine spots, an added feature allows removing all blobs smaller than a user-defined number of pixels, in both single image and batch analyses. b) Defining "regions of interest" (ROI) is also simple, allowing temperature analysis in specific userdefined areas (e.g. an inflammation area, the tail, or any other anatomical area of interest).

a)

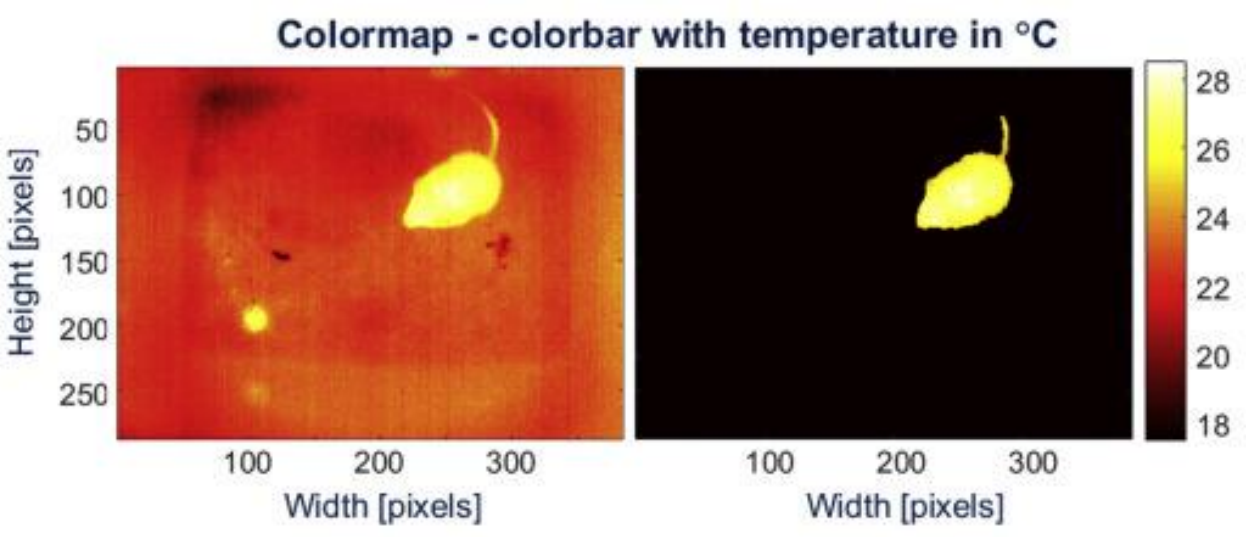

b)
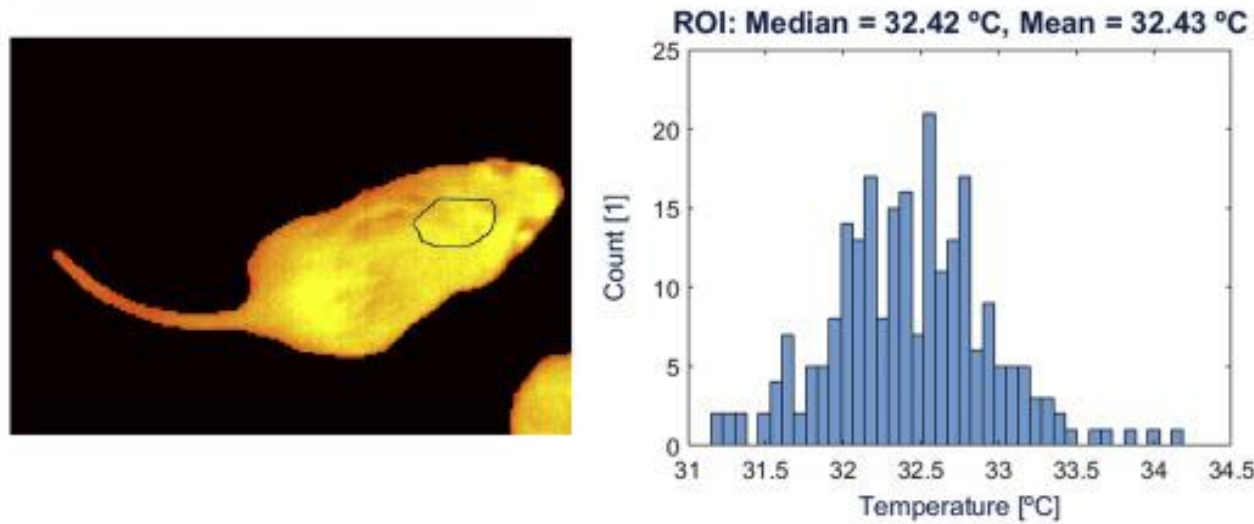

Fig. 4. MBST and subcutaneous temperature following LPS injection. a) The temperature profiles following a high-dose LPS challenge was consistent in all three cages (treatment groups) and between PIT tags and MBST method. b) Temperature profiles combining (mean) the 3 groups of 3 mice $(\mathrm{N}=9)$. The largest mean MBST variation $\left(-4.6^{\circ} \mathrm{C}\right.$ ) was found between $\mathrm{t}=0.33 \mathrm{~h}(20 \mathrm{~min}$ post-LPS injection) and $\mathrm{t}=4.67 \mathrm{~h}$ (280 min post LPSinjection). The largest subcutaneous temperature variation $\left(-5.4{ }^{\circ} \mathrm{C}\right.$, between $\mathrm{t}=0.33 \mathrm{~h}$ and $\left.\mathrm{t}=4.67 \mathrm{~h}\right)$ is an underestimate, since temperatures under $33.0^{\circ} \mathrm{C}$ were under the lower reading range limit of the PIT tag reader, and thus scored as $32.9^{\circ} \mathrm{C}$, for analysis. Asterisks indicate significant changes $(p<0.01$ ) between consecutive timepoints (paired samples t-test, with Holm-Bonferroni correction for multiple comparisons). Bars represent $95 \%$ confidence interval. 
a)

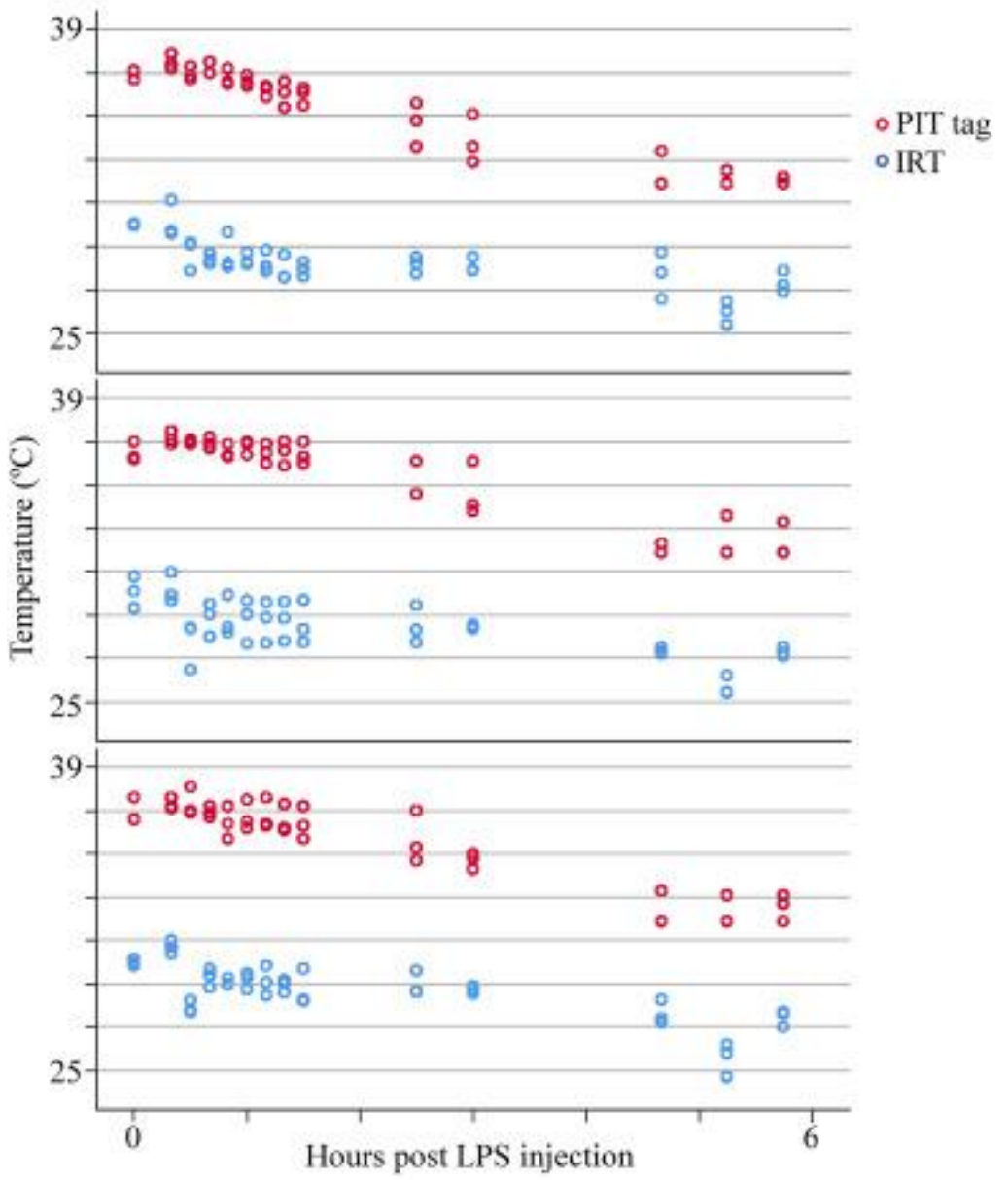

b)

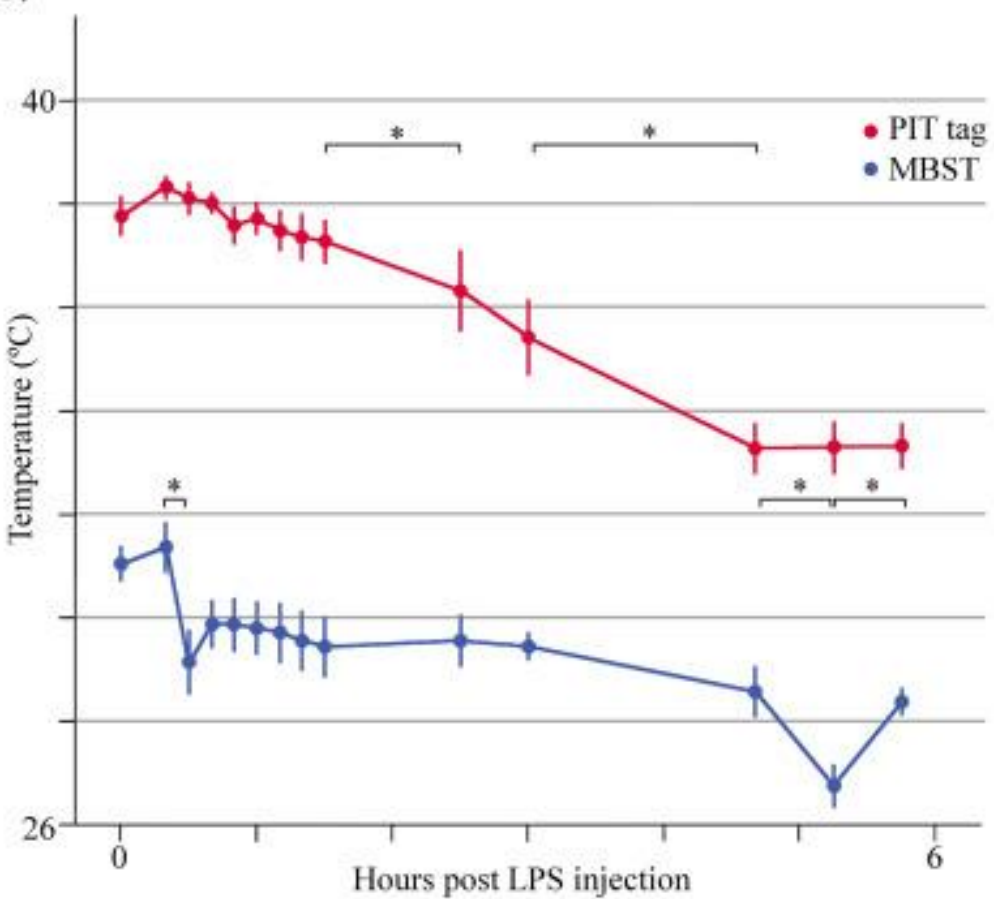

Rua Alfredo Allen, 208 4200-135 Porto 


\section{Discussion}

We developed a software for automatic analysis of thermal images of freely moving mice, and tested it on a mouse model of sepsis-induced hypothermia, comparing its output with the readout from thermo-sensitive PIT-tags. The laborious and time-consuming nature of thermoimage analysis is presently an obstacle to a wider use of thermography in research with laboratory animals. Researchers end up having to rely on general-purpose imaging software (typically associated with camera vendors) which is mainly centred on direct pixel (temperature) readout. Although some allow manually defined regions of interest (ROIs) where statistics can be calculated, these general software lack fundamental tools for efficient measurements of laboratory mice (e.g. segmentation and background subtraction, automatic identification of multiple animals, detection and removal of urine spots, automatic ROIs, batch analysis).

We thus developed ThermoLabAnimal, a computational tool capable of analysing thermal images in .CSV file format, a standard output file used by several commercially available thermal cameras. The software addresses the analysis challenge in two ways. Firstly, through the segmentation algorithm, it automatically separates the pixels in the image that represent the animal from those that represent the background. This removes the need for manually defining the area of analysis, which is time-consuming, as well as prone to inter-user variation. Importantly, instead of collapsing the thermal readout of the segmented pixels into a single statistical measure (such as a mean or median value), the method generates and uses the full histogram of the values to generate a more informative thermal fingerprint. Secondly, the batch analysis function allows automatic and high throughput analysis of all image files located in one folder. We tested software compatibility with both the Thermal Expert camera used in this study (a miniaturized, low-cost thermal camera, a technology that is likely to improve and widen access to IRT technology $[32,33])$ - and the most broadly used thermal camera manufacturer, FLIR (using .CSV files converted by its proprietary software). The software's compatibility can be expanded to other manufacturers, but presently already reads standard. CSV format, with or without manufacturer-specific headers. A comparison between typical general-purpose IRT software and the proposed ThermoLabAnimal software is presented in Table 1.

Table 1

Comparison between general-purpose IRT software (e.g. Fluke SmartView, FLIR Tools, or Testo IrSoft) and ThermoLabAnimal software.

\begin{tabular}{lll}
\hline & Proprietary software & ThermoLabAnimal \\
\hline Compatibility & Compatible with own manufacturer's export formats & FLIR Systems .CSV; Thermo Expert .CSV \\
Batch Analysis & (expandable to other manufacturers'.CSVs) \\
Automatic identification of animals & No & Yes \\
Statistics over a region of interest & No & Yes \\
3D thermal map & No & Yes (freehand regions) \\
Automatic animal body segmentation & No & Yes \\
Price & Variable & Yes \\
& & Free, for version with all features except batch analysis \\
\hline
\end{tabular}

Our protocol and software for assessing mean body surface temperature variation in laboratory animals have the potential to not only improve animal welfare but also the quality of the scientific output, by minimizing interference from handling stress [34,35]. While this is aligned with the 3 Rs principle of Refinement, it can also help further the principle of Reduction, by reducing unwanted inter-individual variability - e.g. from handling stress, sensor position or operator bias - which warrants larger sample sizes for detecting a given effect $[35,36]$. In this experiment, we present data from thermal images of animals placed in a separate cage. We have now developed the software further to allow segmentation of each individual animal, so that future recordings can be done 
without having to remove an animal from its home cage and cage mates. The present version of the software is still unable to identify the same animal across images, thus we are contemplating if it is feasible to add a feature for automatic identification of animals, by using the unique distribution of body surface temperature as an identifier [37], or adding a marker recognizable by the software.

Our results on a mouse model of sepsis-induced hypothermia show that the expected temperature decrease and hypothermia in mice in the first hours following a high-dose LPS injection $[38,39]$ was identifiable by both readout of subcutaneous PIT tags and the automatic estimation of MBST. This decrease was, however, more immediately perceivable in the animals' body surface temperature, which to our knowledge has not previously been monitored for this model. This finding is consistent with the peripheral vasoconstriction expected to follow high LPS dose injection in mice (microcirculatory dysfunction is a central feature of sepsis pathogenesis [40]), which would result in subsequent temperature decrease in peripheral areas due to reduced blood flow. It might also be a physiological response mechanism to maintain core-body temperature $[13,41]$, or a combination of both. A transient small temperature rise was also observable by both methods (although not significant) immediately after intraperitoneal injection of LPS, which is consistent with a quick onset hyperthermic stress response, and in agreement with similar observations in rats [42]. It could, however, also be attributed to an increase of activity following the disturbance of the animals and the removal of the nesting material, or to a putative short-lived fever response following the LPS challenge, immediately before the onset of hypothermia. We also observed a rise in skin temperature resulting from placing the cages over a warm heating pad, although how this affected subcutaneous temperature is uncertain, due to the PIT tags not being able to register temperatures below $33^{\circ} \mathrm{C}$.

It is worth mentioning that the easiness of image analysis brought about by this software allows for greater monitoring frequency, which can be an advantage for many animal studies, especially during short standardized behavioural tests (e.g. open field test). For this particular study, however, in which more interventions other than collecting images - as this was coupled to another experiment - were carried out, this would mean making animals endure prolonged periods under a bright light (and warrant a cage with taller walls) or disturb their microenvironment more frequently.

We found it would not be appropriate to test for correlation between MBST and subcutaneous temperature, even though temperature decrease was observable by the two methods. Firstly, because the PIT tags have a hard lower bound and missed data below $33^{\circ} \mathrm{C}$, which prevented identifying further temperature decreases from $t=4,67 \mathrm{~h}$ onward. Secondly, despite standardised implantation methods, we found great variation in PIT tag position (upon post-mortem examination, these could be found in some animals in the interscapular region, in others on the flank of the animal, and others on the backside), and such variation is a likely contributing factor to the unreliability of PIT tags to inform on core body temperature in vivo despite providing accurate readings in vitro [12]. More accurate information on the relationship between this proxy measure of core body temperature and MBST could be sought in further studies, by fixing the position of the thermo-sensitive transponders, either on the dorsal or abdominal region [43].

Another possibility would be to compare MBST with maximum eye temperature, since it has been found to be a good proxy of other measures of core body temperature in mice [44], as the eyes are supplied by blood from the ophthalmic artery from the brain [44]. However, the relationship between eye temperature and core temperature may be easily affected by peripheral vasoconstriction, either elicited exposure to cold $[45,46]$ or acute stressors $[11,23,47]$. Moreover, we have found it not to be well suited for measuring freely-moving mice, as constant shifts in head position will affect the thermal readout from the eyes. Furthermore, the eyes are often not visible, leading to up to $40 \%$ missing values, even when taking three images per time-point [1]. It is therefore not surprising that others have recommended picking up and restraining mice to place their eyes 
directly in front of the thermal camera for estimating absolute temperature by IRT [44], losing the advantages of contactless measurement.

Perhaps most importantly, there is no 'pure' measure of body temperature, just local temperatures in different parts of the body - internal or external - each of them with its particular bias [48]. Therefore, although in small animals skin temperature is more likely connected with core temperature than in larger species [30], we do not wish to make the claim that MBST can be used as a proxy for mice core body temperature. We are rather proposing that MBST - obtained by means of our software - can be a reliable, non-invasive approach to identify and monitor temperature changes in freely-moving group-housed mice with minimal disturbance. It is however worth mentioning that gathering species-specific thermographic data could contribute to defining standard values for MBST that could be used as a reference value in and of itself.

ThermoLabAnimal is distributed as free software with all its features except for the "batch analysis" mode, which is available at a small fee. The paid full version does not require MATLAB on the user side, and the fee is intended to promote long-term support and improvement of the software. We encourage colleagues to use the software and provide feedback to promote improvements and bug fixes. ThermoLabAnimal software is available on GitHub: https://github.com/ThermoLabAnimal.

\title{
Acknowledgements
}

This work was partially funded by National Funds through FCT - Fundação para a Ciência e a Tecnologia under the project SRecognite Infect-ERA/0003/2015 that also funds Liliana Oliveira. Equipment was partially funded by a UFAW Small Travel and Project Award for Nuno Franco (UFAW Grant 25-2016/2017). Funding also by FEDER - Fundo Europeu de Desenvolvimento Regional funds through the COMPETE 2020 - Operacional Programme for Competitiveness and Internationalisation (POCI), Portugal 2020, and by Portuguese funds through FCT - Fundação para a Ciência e a Tecnologia/Ministério da Ciência, Tecnologia e Ensino Superior in the framework of the project "Institute for Research and Innovation in Health Sciences" (POCI-01-0145-FEDER-007274). Ana Gerós is funded by FCT - Fundacão para a Ciência e a Tecnologia, grant contract SFRH/BD/137385/2018.

We would like to thank Joaquim Gabriel Mendes and Ricardo Vardasca, at the University of Porto Faculty of Engineering, for carrying out the tests with a blackbody radiation source.

\section{Appendix A. Supplementary data}

Supplementary data to this article can be found online at https://doi.org/10.1016/j.physbeh.2019.05.004.

\author{
.
}




\section{REFERENCES}

[1] K. Gjendal, N.H. Franco, J.L. Ottesen, D.B. Sørensen, I.A.S. Olsson, Eye, body or tail? Thermography as a measure of stress in mice, Physiol. Behav. 196 (2018) 135-143.

[2] B. Lecorps, H.G. Rödel, C. Féron, Assessment of anxiety in open field and elevated plus maze using infrared thermography, Physiol. Behav. 157 (2016) 209-216.

[3] C.W. Meyer, Y. Ootsuka, A.A. Romanovsky, Body Temperature Measurements for Metabolic Phenotyping in Mice, Front. Physiol. 8 (2017).

[4] A. Bautista, J.A. Zepeda, V. Reyes-Meza, C. Féron, H.G. Rödel, R. Hudson, Body mass modulates huddling dynamics and body temperature profiles in rabbit pups, Physiol. Behav. 179 (2017) 184-190.

[5] G.J. Tattersall, Infrared thermography: A non-invasive window into thermal physiology, Comparat. Biochem. Physiol. Part A 202 (2016) 78-98.

[6] J.T. Mufford, M.J. Paetkau, N.J. Flood, G. Regev-Shoshani, C.C. Miller, J.S. Church, The development of a non-invasive behavioral model of thermal heat stress in laboratory mice (Mus musculus), J. Neurosci. Methods 268 (2016) 189-195.

[7] J.M. David, A.F. Chatziioannou, R. Taschereau, H. Wang, D.B. Stout, The hidden cost of housing practices: using noninvasive imaging to quantify the metabolic demands of chronic cold stress of laboratory mice, Comparative Med. 63 (2013) 386-391.

[8] J. Adriaan Bouwknecht, B. Olivier, R.E. Paylor, The stress-induced hyperthermia paradigm as a physiological animal model for anxiety: A review of pharmacological and genetic studies in the mouse, Neurosci. Biobehav. Rev. 31 (2007) 41-59.

[9] C. Gordon, Thermal physiology of laboratory mice: defining thermoneutrality, J.Therm. Biol. 37 (2012) 654-685.

[10] A. Marks, D.M.L. Vianna, P. Carrive, Nonshivering thermogenesis without interscapular brown adipose tissue involvement during conditioned fear in the rat, Am. J. Phys. Regul. Integr. Comp. Phys. 296 (2009) R47-R1239.

[11] D.M. Vianna, P. Carrive, Changes in cutaneous and body temperature during and after conditioned fear to context in the rat, Eur. J. Neurosci. 21 (2005) 2505-2512.

[12] J. Hartinger, D. Külbs, P. Volkers, K. Cussler, Suitability of temperature-sensitive transponders to measure body temperature during animal experiments required for regulatory tests, Altex 20 (2002) 65-70.

[13] C.J. Gordon, P.J. Spencer, J. Hotchkiss, D.B. Miller, P.M. Hinderliter, J. Pauluhn, Thermoregulation and its influence on toxicity assessment, Toxicology 244 (2008) 87-97.

[14] T.J. Zethof, J.A. Van Der Heyden, J.T. Tolboom, B. Olivier, Stress-induced hyperthermia in mice: a methodological study, Physiol. Behav. 55 (1994) 109-115.

[15] L.D. Sanford, L. Yang, L.L. Wellman, Telemetry in Mice: Applications in Studies of Stress and Anxiety Disorders. Mood and Anxiety Related Phenotypes in Mice, Springer, 2011, pp. 43-60.

[16] D.B. Morton, P. Hawkins, R. Bevan, K. Heath, J. Kirkwood, P. Pearce, et al., Refinements in telemetry procedures, Lab. Anim. 37 (2003) 261-300.

[17] B.G. Helwig, J.A. Ward, M.D. Blaha, L.R. Leon, Effect of intraperitoneal radiotelemetry instrumentation on voluntary wheel running and surgical recovery in mice, J. Am. Assoc. Lab. Anim. Sci. 51 (2012) 600 JAALAS..

[18] X. Tang, D. Sanford, L. Telemetric recording of sleep and home cage activity in mice, Sleep 25 (2002) 677-685. 
[19] E. Herbut, J. Walczak, Infrared thermography as a method for evaluating the welfare of animals subjected to invasive procedures-a review, Ann. Anim. Sci. 13 (2013) 423-434.

[20] J.S. Church, N.J. Cook, A.L. Schaefer, Recent applications of infrared thermography for animal welfare and veterinary research: everything from chicks to elephants, InfraMation (2009) 215224.

[21] Fabio Luzi, Malcolm Mitchell, Leonardo Nanni Costa, V. Redaelli, Thermography - Current status and advances in livestock animals and in veterinary medicine, Brescia, 9788897562061 Fondazione Iniziative Zooprofilattiche e zootecniche, 2013 ISBN: 9788897562061 https://www.cabdirect.org/cabdirect/abstract/20153288876.

[22] C. Harshaw, J.R. Alberts, Group and individual regulation of physiology and behavior: A behavioral, thermographic, and acoustic study of mouse development, Physiol. Behav. 106 (2012) $670-682$.

[23] N. Ludwig, M. Gargano, F. Luzi, C. Carenzi, M. Verga, Technical note: Applicability of infrared thermography as a non invasive measurements of stress in rabbit, World Rabbit Sci. 15 (2010) 199206.

[24] J. Vadlejcha, I. Knízkovác, K.r. Makovcováa, P. Kuncc, I. Jankovskáa, K. Jandab, et al., Thermal profile of rabbits infected with eimeria intestinalis, Vet. Parasitol. 171 (2010) 343-345.

[25] I. Całkosiński, M. Dobrzyński, J. Rosińczuk, K. Dudek, A. Chrószcz, K. Fita, et al., The use of infrared thermography as a rapid, quantitative, and noninvasive method for evaluation of inflammation response in different anatomical regions of rats, Biomed. Res. Int. 2015 (2015) 9.

[26] M.D. Martinez, M. Ghamari-Langroudi, A. Gifford, R. Cone, E.B. Welch, Automated pipeline to analyze non-contact infrared images of the paraventricular nucleus specific leptin receptor knockout mouse model, SPIE Med. Imaging (2015) 7.

[27] K. Manzano-Szalai, I. Pali-Schöll, D. Krishnamurthy, C. Stremnitzer, I. Flaschberger, E. Jensen-Jarolim, Anaphylaxis imaging: non-invasive measurement of surface body temperature and physical activity in small animals, PLoS One 11 (2016) e0150819.

[28] C. Gilbert, D.J. McCafferty, S. Giroud, A. Ancel, S. Blanc, Private heat for public warmth: how huddling shapes individual thermogenic responses of rabbit pups, PLoS One 7 (2012) e33553.

[29] K.A. Herborn, P. Jerem, R.G. Nager, D.E.F. McKeegan, D.J. McCafferty, Surface temperature elevated by chronic and intermittent stress, Physiol. Behav. 191 (2018) 47-55.

[30] D.J. McCafferty, S. Gallon, A. Nord, Challenges of measuring body temperatures of freeranging birds and mammals, Animal Biotelemet. 3 (2015) 33.

[31] D.R. Powers, K.M. Langland, S.M. Wethington, S.D. Powers, C.H. Graham, B.W. Tobalske, Hovering in the heat: effects of environmental temperature on heat regulation in foraging hummingbirds, R. Soc. Open Sci. 4 (2017).

[32] T. Clausing, Thermography-past, present and future, Badania Nieniszczące I Diagnostyka, 2016, pp. 49-50.

[33] O. Maillot, N. Leduc, V. Atallah, P. Escarmant, A. Petit, S. Belhomme, et al., Evaluation of acute skin toxicity of breast radiotherapy using thermography: results of a prospective single-centre trial, Cancer/Radiothérapie 22 (3) (2018) 205-210.

[34] K. Gouveia, J.L. Hurst, Reducing mouse anxiety during handling: effect of experience with handling tunnels, PLoS One 8 (2013) e66401.

[35] J.D. Bailoo, T.S. Reichlin, H. Würbel, Refinement of experimental design and conduct in laboratory animal research, ILAR J. 55 (2014) 383-391.

[36] R.M.A. Parker, W.J. Browne, The place of experimental design and statistics in the 3 Rs, ILAR J. 55 (2014) 477-485.

(1) 
[37] M. Mazur-Milecka, Thermal imaging in automatic rodent's social behaviour analysis, 13th Quantitative InfraRed Thermography Conference, QIRT Council, Gdańsk, Poland, 2016, pp. 563-569.

[38] H. Saito, E.R. Sherwood, T.K. Varma, B.M. Evers, Effects of aging on mortality, hypothermia, and cytokine induction in mice with endotoxemia or sepsis, Mech. Ageing Dev. 124 (2003) 1047-1058.

[39] R. Blanqué, C. Meakin, S. Millet, C.R. Gardner, Hypothermia as an indicator of the acute effects of lipopolysaccharides: comparison with serum levels of IL1 $\beta$, IL6 and TNF $\alpha$, General Pharmacol. 27 (1996) 973-977.

[40] P.R. Baver, Microvascular responses to sepsis: clinical significance, Pathophysiology 8 (2002) $141-148$.

[41] J.M. Overton, Phenotyping small animals as models for the human metabolic syndrome: thermoneutrality matters, Int. J. Obesity (2005) 34 (Suppl. 2) (2010) $S_{53}-S_{5} 8$.

[42] M.C. Almeida, A.A. Steiner, L.G. Branco, A.A. Romanovsky, Cold-seeking behaviour as a thermoregulatory strategy in systemic inflammation, Eur. J. Neurosci. 23 (2006) 3359-3367.

[43] W. Kort, J. Hekking-Weijma, M. Tenkate, V. Sorm, R. VanStrik, A microchip implant system as a method to determine body temperature of terminally ill rats and mice, Lab. Anim. 32 (1998) 260269.

[44] B. Vogel, H. Wagner, J. Gmoser, A. Wörner, A. Löschberger, L. Peters, et al., Touchfree measurement of body temperature using close-up thermography of the ocular surface, Methods $\mathrm{X}_{3}$ (2016) 407-416.

[45] G. Piccione, M. Gianesella, M. Morgante, R. Refinetti, Daily rhythmicity of core and surface temperatures of sheep kept under thermoneutrality or in the cold, Res. Vet. Sci. 95 (2013) 261-265.

[46] F. Vannetti, S. Matteoli, L. Finocchio, F. Lacarbonara, A. Sodi, U. Menchini, et al., Relationship between ocular surface temperature and peripheral vasoconstriction in healthy subjects: A thermographic study, Proceedings of the Institution of Mechanical Engineers, Part H 228 (2014) 297-302.

[47] K.A. Herborn, J.L. Graves, P. Jerem, N.P. Evans, R. Nager, D.J. McCafferty, et al., Skin temperature reveals the intensity of acute stress, Physiol. Behav. 152 (2015) 225-230.

[48] D.L. Vianna, P. Carrive, Visualisation of thermal changes in freely moving animals, in: E. Badoer (Ed.), Visualization Techniques, Humana Press, 2012, pp. 269-281.

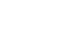

